\title{
Correction to: Mathematical Modelling of Alternative Pathway of Complement System
}

\section{Suruchi Bakshi, et al. [full author details at the end of the article]}

Published online: 18 December 2021

(c) Society for Mathematical Biology 2021

\section{Correction to: Bulletin of Mathematical Biology (2020) 82:33 https://doi.org/10.1007/s11538-020-00708-z}

The original version of the article unfortunately contained mistakes.

One of the schematics (Figure 3) shows a mismatch with the corresponding model equations (C.1). This was due to an error in the schematic.

Furthermore, there was a typo in the value of a parameter $d_{4}$ in Table 1 . The correct value is 0.00016 per min-which was used in the simulations.

We acknowledge Biomodels (https://www.ebi.ac.uk/biomodels/) for bringing this to our notice. These changes do not impact the results and conclusions presented in the paper.

These two discrepancies have been corrected in this correction.

Corrected Figure 3 schematic: 


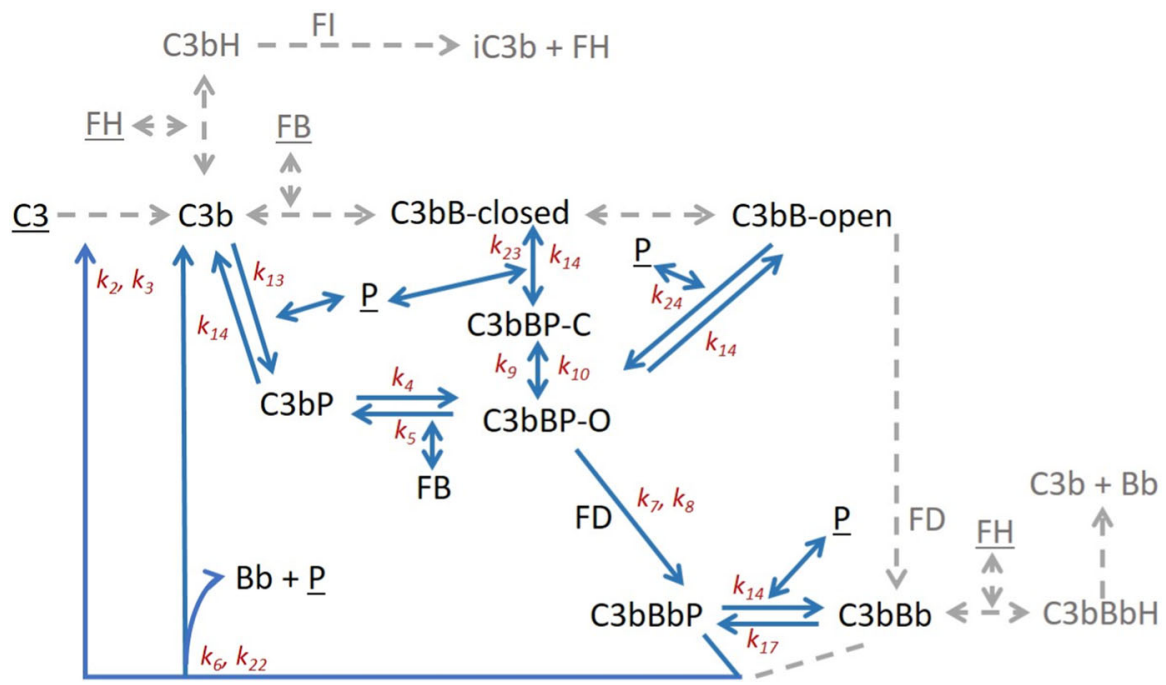

Fig. 3 Schematic representation of the reactions in the properdin model. Properdin model is an expansion of the minimal model. The components and reactions from the minimal model, which do not bind properdin, are greyed out and simplified for better clarity. However, these reactions are modeled exactly as in the minimal model. Underlined components are subject to synthesis and degradation reactions, which are included in this model but excluded from the diagram for clarity. Reaction rate constants are indicated on the respective reaction arrows.

Publisher's Note Springer Nature remains neutral with regard to jurisdictional claims in published maps and institutional affiliations.

\section{Affiliations}

Suruchi Bakshi ${ }^{1,2}$ (1) $\cdot$ Fraser Cunningham ${ }^{3} \cdot$ Eva-Maria Nichols $^{3}$. Marta Biedzka-Sarek ${ }^{3}$. Jessica Neisen ${ }^{3}$. Sebastien Petit-Frere ${ }^{3}$. Christina Bessant ${ }^{3}$. Loveleena Bansal ${ }^{4}$. Lambertus A. Peletier ${ }^{5}$. Stefano Zamuner $^{6}$. Piet H. van der Graaf ${ }^{1,7}$

$凶$ Suruchi Bakshi

suruchi.bakshi@certara.com

Fraser Cunningham

fraser.x.cunningham@gsk.com

Eva-Maria Nichols

eva-maria.x.nichols@gsk.com

Marta Biedzka-Sarek

marta.biedzka-sarek@roche.com

Jessica Neisen

jessica.x.neisen@gsk.com

Sebastien Petit-Frere

sebastien.x.petit-frere@gsk.com 
Christina Bessant

christina.m.bessant@gsk.com

Loveleena Bansal

loveleena.x.bansal@gsk.com

Lambertus A. Peletier

peletier@math.leidenuniv.nl

Stefano Zamuner

stefano.6.zamuner@gsk.com

Piet H. van der Graaf

Piet.vanderGraaf@certara.com

1 Division of Systems Biomedicine and Pharmacology, LACDR, Leiden University, P.O.

Box 9502, 2300 RA Leiden, The Netherlands

2 Certara QSP, 4818 SJ Breda, The Netherlands

3 Cytokine, Chemokine and Complement DPU, Immunoinflammation TA Unit, GSK, Stevenage, UK

4 Computational and Modelling Sciences, Platform Technology Sciences, GSK, Collegeville, Pennsylvania, USA

5 Mathematical Institute, Leiden University, P.O. Box 9512, 2300 RA Leiden, The Netherlands

6 Clinical Pharmacology, Modelling and Simulation, GSK, Stevenage, UK

7 Certara QSP, Canterbury CT2 7FG, UK 